\title{
Body Image During Quarantine; Generational Effects of Social Media Pressure on Body Appearance Perception
}

\author{
Abrevaya, Sofia (sabrevaya@conicet.gov.ar) \\ National Scientific and Technical Research Council; Godoy Cruz 2290, C1425 CABA, Argentina \\ Instituto de Neurociencia Cognitiva y Traslacional; Marcelo Torcuato de Alvear 1632, C1021 CABA, Argentina \\ Speranza, Trinidad B. (trinidadsperanza@uca.edu.ar) \\ National Scientific and Technical Research Council; Godoy Cruz 2290, C1425 CABA, Argentina
}

Centro de Investigaciones en Psicología y Psicopedagogía, Universidad Católica Argentina; Alicia Moreau de Justo 1800, C1107 CABA, Argentina

\section{Perez Cano, María de Guadalupe (mguadalupepc@gmail.com)}

Centro de Investigaciones en Psicología y Psicopedagogía, Universidad Católica Argentina; Alicia Moreau de Justo 1800, C1107 CABA, Argentina

\section{Ramenzoni, Verónica C. (veronicaramenzoni@uca.edu.ar)}

National Scientific and Technical Research Council; Godoy Cruz 2290, C1425 CABA, Argentina

Centro de Investigaciones en Psicología y Psicopedagogía, Universidad Católica Argentina; Alicia Moreau de Justo 1800, C1107 CABA, Argentina

\begin{abstract}
One of the consequences of the pandemic is that throughout 2020 virtual interactions largely replaced face-to-face interactions. Though there are few studies of how social media impacts body image perception across genders, research suggests that socializing through a virtual self-body image might have distinct implications for men and women. On an online study, we examined whether type of social pressure and body-ideal exert distinct pressures on members of the X, Y, and $\mathrm{Z}$ generations. Results showed media pressure affected body image satisfaction significantly more than other kinds of social pressure across genders and generations, with young males reporting a higher impact compared to older males. Males experienced more pressure to be muscular and women to be thin, especially for the younger generation.Future research should focus on social media as a potential intervention tool for the detection and prevention of body image disorders in both young female and male adults.
\end{abstract}

Keywords: Social Pressure; Social Media; Body Image; Traditional Media. terms or keywords; kindly use a semicolon; between each term

\section{Introduction}

Body image is a complex construct that affects the cognitive attitudes and evaluations that we make of ourselves; it determines how we perceive our 'look' and how we relate to other people through how we experience their 'looks'. As a construct, it does not reflect the physical characteristics of the body but rather it is built on a systematic bias in the perception of anthropometric body dimensions (Sadibolova et al., 2019; Linkenauger et al., 2015) that, in turn, recruits cognitive-affective and social perception processes (Linkenauger et al., 2017). The body image changes and adapts as the body changes (e.g., during adolescence or with large weight fluctuations) and its open to the external social influences that range from friends and family to the cultural forces such as the body ideal promoted by the media. These influences affect self-perception, the evaluative judgments people make about their own body image in relation to the environment. When the discrepancy between evaluative judgments about the body and its physical characteristics is too great, it results in a dysfunctional body image or body image disorder (Pitron, Alsmith, \& Vignemont, 2018). The ability to detect the degree of perceived body image distortion early in risk populations is critical and may be the most successful way of reducing their incidence in the general population.

In the last two decades, changes in the forms of social interaction afforded by digital tools have introduced a new medium through which the social environment influences body image formation and the emergence of related disorders. Social media refers to "a group of internet-based applications that build on the ideological and technological foundations of Web 2.0, and that allow the creation and exchange of user-generated content" (Kaplan \& Haenlein, 2010). Social networks are a very popular phenomenon among Internet users in Argentina, where more than 90\% participate in one or more of these (Carrier, 2016; Ravalli \& Paoloni, 2016). Among internet users, younger people are the ones who are connected the longest. The internet is a central and indispensable element in the lives of adolescents and young adults more so that of older adults' (Delfino, 2017; 
Dogliotti, 2020). According to an Argentinian study, people use the internet for two main purposes: social engagement (eg, chatting with friends) and utilitarian use (eg, reading news). This study and other local studies found clusters, made up of mostly young people, who made intensive use of the Internet and social networks (i.e., more than 4 hours per day); their activity consisted largely on sharing photos and moods (Solano \& Lupano Perugini, 2019). Within internet users, communication and the use of social networks is associated with body image problems, disordered eating behaviors, and changes in the body (such as dietary restriction and muscle development) in younger generations (Rodgers et al., 2020).

A generation of people is defined by a set of historical events and related phenomena that distinguish a cultural gap (Parry \& Urwin, 2011). A relatively recent Pew Research study (Taylor \& Keeter, 2010) defined five generations: the silent generation (those born between 1922 and 1945), the baby boomers (born from 1946 to 1964); Generation X (born from 1965 to 1977); and millennials (born from about 1977 to 1993) and Generation Z, youth born in the mid-1990s through the late 2010s. Younger generations that are constantly exposed to computers and other technologies are considered to "digital natives" (Bennett, 2012), while older generations are considered "digital immigrants" that have to learn to use these new tools in adulthood (Prensky, 2001). In particular, Generation $\mathrm{Z}$ individuals are considered true digital natives because they never experienced life before the Internet. As a consequence, they have become accustomed to interacting and communicating in a world that is connected at all times (Turner, 2015).

For the most part studies have looked at social media as a new force that has a singular influence but have not directly compared the pressure it exerts on body image for both genders across generations with long established sources of pressure (i.e., family, peers, traditional media) (Fardouly \& Vartanian, 2016). A recent study found that that younger adults use Facebook more frequently and are significantly more emotionally impacted by the site than older adults (Hayes, van Stolk-Cooke, \& Muench, 2015). However, a cross-sectional study found that having and using a Facebook profile is associated with poorer body satisfaction for both men and women, and across all ages (Stronge et al., 2015). Recently, the worldwide quarantine forced us to be alone with ourselves, reporting negative psychological effects (Brooks et al., 2020). Social media exposure has been positively associated with prevalence of mental health problems during the COVID-19 outbreak. Social media related content has conveyed strong fears of gaining weight or becoming "fat" (Pearl, 2020). Social distancing has changed how we relate to our bodies, not only by being isolated from social pressures but also through changes in eating patterns as well as body presentation. The new reality brought up by social isolation during the pandemic that has limited human contact for the most part to digitally mediated social interactions highlight the importance of comprehensive studies of body satisfaction social media and other influences on body image formations. In Argentina, a significant percentage of women experienced negative changes related to their diet and their body image during quarantine, including behaviors considered risky for the development of eating disorders (Sanday, 2020).

\section{Current Study}

The current study examined whether social media pressure is different to other kinds of social pressure and has a distinct effect on people's body image depending on their generation and gender. To assess this issue, we administered a modified version of the Sociocultural Attitudes Towards Appearance Questionnaire SATAQ-4R that included questions about social media use to a wide sample of individuals (18 to $55 \mathrm{yrs}$ of age) of both genders. The study was conducted online 3 months into a strict quarantine that prevented all types of social distancing (May-June 2020). The main hypothesis guiding this project was that social media pressures on body appearance perception - not included in the SATAQ-4Rcould have a distinct influence compared to other kinds of pressure and in particular traditional media pressure. We expected that social media would affect people differently depending on their age (i.e., the generation they belonged to) and that, in line with previous work, external pressures would have a more pronounced effect on women compared to men.

\section{Methods}

\section{Participants}

Between May and June 2020, 957 subjects (528 females, 419 males, 11 other gender; $M=30.42$ years old, $S D=11.20$ years) answered an online survey that included an initial set of demographic and social media use questions and the modified version of the SATAQ-4. Participants were recruited through online ads and posts in Facebook and Instagram, and through WhatsApp groups of college students. Before the beginning of the survey participants gave their informed consent to participate in the study. Informed consents followed the norms of the declaration of Helsinki and were adapted to be administered online. All respondents completed high school education or higher, and were born and lived in Argentina, they came from different cities across the country, therefore all questionnaires were in Spanish. A number of respondents were excluded from the final sample, due to their very small representation in the final sample, were transgender, foreigners, underage and over fifty-five, and those ones that failed to answer all required questions. The sample submitted for analyses included 827 respondents grouped according to their age in three generations: Generation X (40-55-yrs-old); Generation Y (26-39-yrs.old), and Generation Z (18-25-yrs.-old). Table 1 includes all demographic data for the relevant variables measured before the administration of the modified version of SATAQ-4.

Table 1: Age and body mass index (BMI) mean (M) and standard deviation (SD), age range, gender and social network sites daily usage (SNS use) for the 3 generations (Gen).

\begin{tabular}{llll} 
Gen Gender & Age & BMI & $\begin{array}{l}\text { SNS } \\
\text { Use }\end{array}$ \\
\hline
\end{tabular}




\begin{tabular}{|c|c|c|c|c|c|}
\hline $\begin{array}{c}\mathrm{Z} \\
(18-25 \\
\text { yrs old })\end{array}$ & 189 & 177 & $\begin{array}{c}21-67 \\
(1.98)\end{array}$ & $\begin{array}{r}23.84 \\
(12.93)\end{array}$ & $\begin{array}{r}4,08 \\
(0.84)\end{array}$ \\
\hline $\begin{array}{c}\mathrm{Y} \\
(26-39 \\
\text { yrs-old) }\end{array}$ & 213 & 138 & $\begin{array}{c}31,45 \\
(3.83)\end{array}$ & $\begin{array}{c}24,45 \\
(4.37)\end{array}$ & $\begin{array}{r}3,40 \\
(0.94)\end{array}$ \\
\hline $\begin{array}{c}\mathrm{X} \\
(40-55 \\
\text { yrs old })\end{array}$ & 65 & 51 & $\begin{array}{c}45,68 \\
(4.53)\end{array}$ & $\begin{array}{c}25,88 \\
(4.72)\end{array}$ & $\begin{array}{r}3,39 \\
(0.96)\end{array}$ \\
\hline
\end{tabular}

\section{Questionnaires}

A demographic and social media use questionnaire was developed and the Sociocultural Attitudes Towards Appearance Questionnaire-4-Revised (SATAQ-4R) was translated to Spanish (Schaefer, 2017), based on the Argentinian validation of SATAQ-3 (Murawski et al., 2015) and the Spanish validation (Llorente et al., 2015). Demographic questions asked about age, sex, gender, education, physical activity, weight, height, birthplace, residence place, chronic disease, eating disorders and, kind and number of people they cohabited with (family, roommates, etc.). Social media use questions asked which SNS they used, how often, what type of content they uploaded, whether they looked at their own profile, if their personal information was accurate in the cites (i.e., not edited or modified), how many online friends did they knew outside of social media, and what were their main purposes for using it. Questions were sourced from a combination of wellknown demographic and social media questionnaires (Andreassen, 2012; Jenkins-Guarnieri, Wright, \& Johnson, 2013) and updated to include the most commonly used social media platforms. Following these questions, participants completed a modified online version of the female or male version of the SATAQ-4R scale (Schaefer, 2017). The SATAQ-4R-Female is a 31-item scale encompassing seven factors: (1) Internalization-Thin/Low Body Fat, (2) Internalization-Muscular, (3) Internalization-General Attractiveness, (4) Pressures-Family, (5) Pressures-Peers, (6) Pressures-Media, and (7) Pressures-Significant Others. The SATAQ-4R-Male is a 28 -item scale that encompasses the same seven factors but contains eight additional items. Each item is rated on a five-point Likert scale ranging from 1 ("definitely disagree") to 5 ("definitely agree"). Per the main goal of this study, we added an eighth factor: (8) PressuresSocial Media. The items added were based on the items from the Pressures-Media factor; questions were identical for both factors but for replacing the wording 'media' for 'social media'. These items were added at the end of the female and male scale so as not to bias the expected responses to the SATAQ-4R.

\section{Analyses}

In order to evaluate the relationship between type of social pressure (family, peer, significant other, media, and social media), generation (X, Y, and $\mathrm{Z}$ ), and gender (male and female), we performed a three-way repeated measures Repeated Measures ANOVA and follow up factorial ANOVAs for each type of pressure and each type of bodyideal were performed with gender and generation as factors

\section{Results}

A significant three-way interaction effect was found between gender, generation and type of social pressure (see Figure 1). ANOVA performed on each type of pressure with generation and gender as factors showed: no significant effects for significant other pressure $\left(F(2,827)=2.30, p=0.10, \eta_{p}{ }^{2}=\right.$ $.005)$, a distinct effect of family pressure for each gender $(F$ $\left.(1,827)=22.77, p=0.00, \eta_{p}{ }^{2}=.003\right)$ with females experiencing more pressure than males regardless of their age $(p=0.00)$ and an effect of peer pressure depending on generation and gender $\left(F(2,827)=11.51, p=0.00, \eta_{p}{ }^{2}=\right.$ $.027)$. Post-hoc analyses showed that comparing genders in generation $\mathrm{Z}$, male respondents experience more pressure than females' respondents. And comparing between generation $\mathrm{Z}$ and $\mathrm{Y}$, younger respondents experience more pressure than older respondents. Analyses of traditional media showed a significant effect between gender and generation $\left(F(2,827)=3.73, p=0.02, \eta_{p}{ }^{2}=.008\right)$. As for gender, post-hoc analysis showed that females experience more pressure than males $\left(F(1,827)=91.96, p=0.00, \eta_{p}{ }^{2}=\right.$ $.010)$. As for generation, there's no difference in females. However, $\mathrm{Z}$ males experience more pressure than $\mathrm{X}$ males $(p$ $<0.00$ ). Analyses of social media showed no significant effect of interaction for gender and generation. However, there were differences between each factor individually: females are the ones who experience the most pressure from social media $\left(F(1,827)=82.85, p=0.00, \eta_{p}{ }^{2}=.009\right)$ and younger generations are the ones who experienced most pressure $\left(F(2,827)=10.88, p=0.00, \eta_{p}{ }^{2}=.002\right)$.
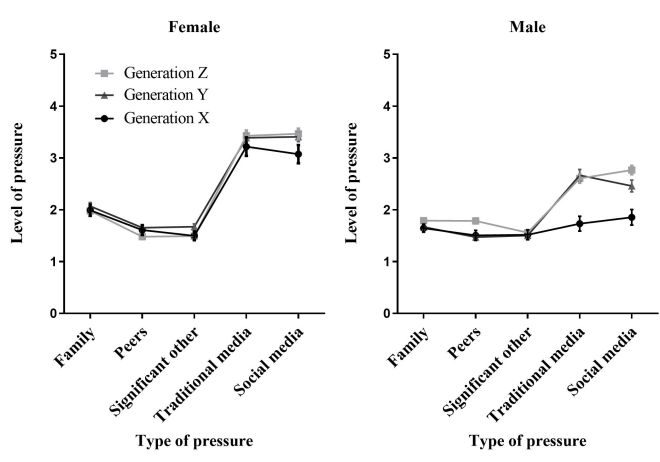

Figure 1: Three-way repeated measures analysis of variance with effect sizes and powers, comparing type of pressure, gender and generation.

Moreover, a significant three-way interaction effect was found between gender, generation and pressure of body-ideal experienced (see Figure 2). ANOVA performed on each type of body-ideal with generation and gender as factors. The 
results showed: no significant interaction in generation $X$; in generation Y significant effects of gender $(F(1,698)=36.61$, $p=0.00)$, type of body-ideal were found $(F(2,698)=19.93$, $p=0.00)$ and the interaction between them $(F(2,698)=$ $26.23, \mathrm{p}=0.00)$. Post-hoc analysis showed that comparing females and males, there were differences between each type of ideals; Y males had no differences between type of bodyideal, however $\mathrm{Y}$ females experimented more pressure in the body-ideal of General Attractiveness. Lastly, in Generation Z there were significant effects of gender $(F(1,728)=35.25, p$ $=0.00)$ and the interaction between gender and type of bodyideal $(F(2,728)=95.75, p=0.00)$. Post-hoc analysis showed that comparing females and males, there were differences between each type of ideals; in $\mathrm{Z}$ males experienced the body-ideal of Muscularity with more pressure compared to the other two types of body-ideals. On the other hand, in Z females General Attractiveness is the body-ideal experienced with more pressure, followed by Low Body Fat and Muscularity.
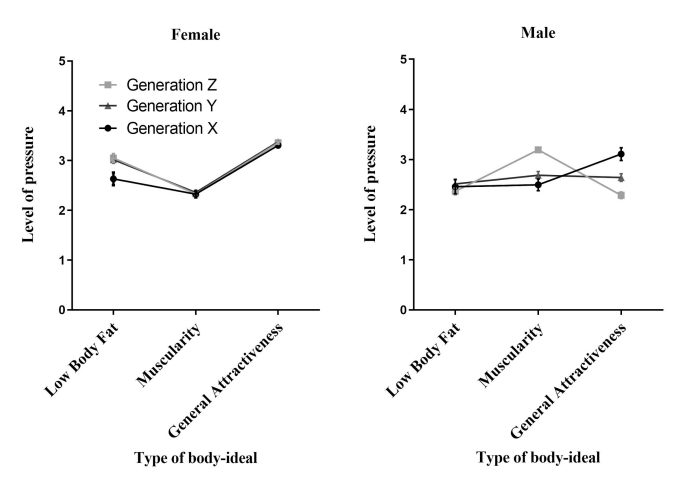

Figure 2: Three-way repeated measures analysis of variance with effect sizes and powers, comparing type of body-ideal, gender and generation.

\section{Discussion}

The aim of this project was to assess how, during a pandemic that forces individuals to abstain from face-to-face interactions and engage in social interactions virtually, different social pressures and specifically social media influence the body image. This study is the first to evaluate different sources of social influence that include social media on both genders and across age groups. A modified version of the SATAQ-4 (Schaefer, 2017) was used to evaluate social pressures and relationship to different types of body ideals.

Recently the SATAQ-4, the most used test to evaluate social pressure on appearance, has been updated (Schaefer, 2017). However, this new version does not differentiate between social networking sites and traditional media. No other research with this questionnaire has evaluated differences between generations (or age related) or social media use. Some studies have found cultural differences (Burnette et al., 2020), appearance pressures and ideals extend beyond thinness or muscularity. And beyond this questionnaire, research on body image rarely evaluates older people, and specifically men of all generations. We sought to assess sources of social influence in a population including three generations (X, Y and $\mathrm{Z}$ ), in both female and male, and adding questions at the end of the SATAQ- $4 \mathrm{R}$ that specifically asked for social media influence.

Overall significant differences were found between generations and genders for traditional media and social media. Both types of media pressure exerted a similarly high degree of perceived social pressure on appearance, higher than any other type of pressure (family, peers and significant others). While no significant differences were found between traditional and social media, social media did not impact each male generation in the same way, while traditional media had a more uniform effect on both men and women of all ages. Significant other pressure showed no differences for either gender, or generations. Family pressure was higher for females but showed no differences within generations. Finally, peer pressure influence was only significantly different within generations. Furthermore, regarding type of body-ideal results, it was demonstrated that younger males (generation Z) experienced the body-ideal of Muscularity with more pressure. As for younger females ( $\mathrm{Z}$ generations) General Attractiveness is the body-ideal experience with more pressure, followed by Low Body Fat and Muscularity. In line with previous studies (Grogan, 2016; Thompson \& Stice, 2001), men tend to aspire for a muscular body (capable of becoming muscular dysmorphia disorder) and women aspire for a thin body, and "attractive" in general (capable of resulting in a possible eating disorder).

The main interest of this study was to evaluate whether a fifth type of social pressure on physical appearance was relevant to consider, considering gender and generational differences. Even though we found a significant interaction effect between genders and generations, and within type of pressure, this effect was only present between generations when comparing only traditional and social media. We did expect lower perceived pressure from older males from the media, but we expected a difference in type of media pressure within generations. In this regard we have two possible interpretations. First of all, currently traditional media has an online presence, and this is a present quality for younger generations that are digital natives, therefore being difficult to differentiate both. Secondly, the questionnaire does not specify the difference between both: for example, talking about Facebook and Instagram for social media, and television and journals for traditional media. Social influence on appearance regarding peers did not differentiate gender or generations. This constituted an unexpected result, particularly for younger generations, where social media had a higher influence than other types of pressure (such as significant others and family) and where the differences were significant between generations. However, due to only including adults in the $\mathrm{Z}$ generation, perhaps the effect of peers' importance in teenage years was diluted (De Vries et al., 2016; Kenny et al., 2017; Chua \& Chang, 2016). Nevertheless, this constitutes a relevant result, that could indicate that media (aside from being traditional or social) exerts influence on younger generations through social comparison of non-peers, such as celebrities (Brown \& Tiggemann, 2020; Brown \& Tiggemann, 2016; Ho, Lee, \& Liao, 2016). 
Regarding the limitations of our research, we consider that we could have had a bigger sample on the male population. Even though the sample is not small, and its size was considered on the statistical analysis, there are more published works on the topic on women, even the original validation of the test had a bigger sample in women than men. Furthermore, due to sample size limitations, some groups were excluded, in particular transgender and older people. Previous research has shown body dissatisfaction in the former (Brewster et al., 2019; McClain \& Peebles, 2016; McGuire et al., 2016; Van de Grift et al., 2016), but none has addressed particularly social media influence in this group. Also considering the significant difference between generations, if we had added the boomer one, it would illuminate whether the tendency to see less impact of media in appearance is maintained for older people.

On the other hand, we performed the data collection during the quarantine in Argentina due to COVID-19, a time where the use of social media in general has increased (Cellini et al., 2020). A follow-up post-pandemic should be done in order to see if these differences are maintained. In addition, we are aware that the SATAQ-4R evaluates other factors that influence pressure on appearance, such as InternalizationMuscular. Concerning physical activity, we asked in the sociodemographic questionnaire if they practice any, how often, and what type of activity they do. Just as the use of social networks could have been affected by the quarantine, the same could have happened with the frequency of physical activity. And specifically, taking into account the Argentinian population, running was prohibited the first 3 months of isolation. It is for this reason, and because of the lack of control that we could access this information, that we decided not to evaluate the relationship that exists between physical activity, gender and generations. In particular, we decided to focus on studying the potential origins of body image disorders (that is, the pressures) and not so much on their consequences (that is, the ideal of beauty). It would be interesting to be able to evaluate in future studies the incidence of physical activity with genders and generations.

Future research should focus on validating the test for the Argentine population, including the last questions regarding social media, and in particular, in teenagers. Considering our results, it constitutes a clear goal to deepen these results in younger people. Our results showed that BMI and social media use functioned as predictors of social influence difference between genders and generations. Also, considering the fact that social media use has been associated with mental health issues (Barry et al., 2017; O'Reilly et al., 2018), eating (Mabe, Forney, \& Keel, 2014) and body image disorders (Perloff, 2014; Rodgers \& Melioli, 2016), we consider that studying population with these pathologies is a matter of importance. Finally, this study only included people from Argentina, and considering previous studies that found differences between cultures in body ideal internalization (Ricciardelli et al., 2007; Lee et al., 2014), a multicenter study comparing different cultures would be of interest.

\section{Conclusions}

Over the past two decades, virtual interactions have become a significant means of socialization. Never has this been more evident than during the current covid-19 pandemic. In this study we find that interacting with a virtual self-body has profound effects on how people of different genders and generations perceive their body image. These effects are more pronounced for younger generations and specifically younger males. This finding highlights the role of social media in our daily lives and how our cognitive system adapts to it. In the future, it would be important to take into consideration how social media tools might affect individuals distinctly based on their gender and age and whether they can be used to promote realistic body images, encourage healthier behaviors, and help ameliorate their negative effects on how we construe our body image.

\section{References}

Andreassen, C. S., Torsheim, T., Brunborg, G. S., \& Pallesen, S. (2012). Development of a Facebook addiction scale. Psychological reports, 110(2), 501-517.

Barry, C. T., Sidoti, C. L., Briggs, S. M., Reiter, S. R., \& Lindsey, R. A. (2017). Adolescent social media use and mental health from adolescent and parent perspectives. Journal of adolescence, 61, 1-11.

Bennett, S. (2012). Digital natives. Encyclopedia of cyber behavior (vol. 1, pp. 212-219). United States: IGI Global.

Brewster, M. E., Velez, B. L., Breslow, A. S., \& Geiger, E. F. (2019). Unpacking body image concerns and disordered eating for transgender women: The roles of sexual objectification and minority stress. Journal of counseling psychology, 66(2), 131.

Brooks, S. K., Webster, R. K., Smith, L. E., Woodland, L., Wessely, S., Greenberg, N., \& Rubin, G. J. (2020). The psychological impact of quarantine and how to reduce it: rapid review of the evidence. The Lancet.

Brown, Z., \& Tiggemann, M. (2016). Attractive celebrity and peer images on Instagram: Effect on women's mood and body image. Body image, 19, 37-43.

Brown, Z., \& Tiggemann, M. (2020). A picture is worth a thousand words: The effect of viewing celebrity Instagram images with disclaimer and body positive captions on women's body image. Body Image, 33, 190198.

Burnette, C. B., Boutté, R. L., Sosnowski, D. W., \& Mazzeo, S. E. (2020). Measurement invariance of the SATAQ-4 in Black and White undergraduate women. Body Image, 32, 85-93.

Carrier, E. (2016, June 24). Redes Etarias. Retrieved from: https:/comentarios.info/index.php/2016/06/24/redesetarias/

Cellini, N., Canale, N., Mioni, G., \& Costa, S. (2020). Changes in sleep pattern, sense of time and digital media use during COVID-19 lockdown in Italy. Journal of Sleep Research, e13074.

Chua, T. H. H., \& Chang, L. (2016). Follow me and like my beautiful selfies: Singapore teenage girls' engagement in self-presentation and peer comparison on 
social media. Computers in Human Behavior, 55, 190197.

Delfino, G., Sosa, F., \& Zubieta, E. (2017). Internet usage in Argentina: Gender and age as variables associated to the digital divide. Investigación \& desarrollo, 25(2), 100-123.

de Vries, D. A., Peter, J., de Graaf, H., \& Nikken, P. (2016). Adolescents' social network site use, peer appearancerelated feedback, and body dissatisfaction: Testing a mediation model. Journal of youth and adolescence, 45(1), 211-224.

Dogliotti, C., González-Insua, F., Botero, C., \& Delfino, G. (2020). Uso y frecuencia de conexión a internet y bienestar subjetivo en jóvenes argentinos. PSICOLOGÍA UNEMI, 4(7), 74-87.

Fardouly, J., \& Vartanian, L. R. (2016). Social media and body image concerns: Current research and future directions. Current opinion in psychology, 9, 1-5.

Grogan, S. (2016). Body image: Understanding body dissatisfaction in men, women and children. Taylor \& Francis.

Hayes, M., van Stolk-Cooke, K., \& Muench, F. (2015). Understanding Facebook use and the psychological effects of use across generations. Computers in Human Behavior, 49, 507-511.

Ho, S. S., Lee, E. W., \& Liao, Y. (2016). Social network sites, friends, and celebrities: The roles of social comparison and celebrity involvement in adolescents' body image dissatisfaction. Social Media + Society, 2(3), 2056305116664216.

Jenkins-Guarnieri, M. A., Wright, S. L., \& Johnson, B. (2013). Development and validation of a social media use integration scale. Psychology of popular media culture, 2(1), 38.

Kaplan, A., \& Haenlein, M. (2010). Users of the world, unite! The challenges and opportunities of social media. Business Horizons, 53, 59-68.

Kenny, U., O’Malley-Keighran, M. P., Molcho, M., \& Kelly, C. (2017). Peer influences on adolescent body image: friends or foes?. Journal of Adolescent Research, 32(6), 768-799.

Lee, H. R., Lee, H. E., Choi, J., Kim, J. H., \& Han, H. L. (2014). Social media use, body image, and psychological well-being: A cross-cultural comparison of Korea and the United States. Journal of health communication, 19(12), 1343-1358.

Linkenauger, S. A., Kirby, L. R., McCulloch, K. C., \& Longo, M. R. (2017). People watching: Ther perception of the relative body proportions of the self and others. Cortex, 92, 1-7.

Llorente, E., Gleaves, D. H., Warren, C. S., Pérez-deEulate, L., \& Rakhkovskaya, L. (2015). Translation and validation of a spanish version of the sociocultural attitudes towards appearance questionnaire-4 (SATAQ4). International Journal of Eating Disorders, 48(2), 170175.

Mabe, A. G., Forney, K. J., \& Keel, P. K. (2014). Do you "like" my photo? Facebook use maintains eating disorder risk. International Journal of Eating Disorders, 47(5), 516-523.

McClain, Z., \& Peebles, R. (2016). Body image and eating disorders among lesbian, gay, bisexual, and transgender youth. Pediatric Clinics, 63(6), 1079-1090.

McGuire, J. K., Doty, J. L., Catalpa, J. M., \& Ola, C. (2016). Body image in transgender young people: Findings from a qualitative, community based study. Body Image, 18, 96-107.

Murawski, B., Elizathe, L., Custodio, J., \& Rutsztein, G. (2015). Argentine validation of the Sociocultural Attitudes Towards Appearance Questionnaire-3. Revista Mexicana de Trastornos Alimentarios, 6(2), 73-90. https://doi.org/10.1016/j.rmta.2015.09.001

O'Reilly, M., Dogra, N., Whiteman, N., Hughes, J., Eruyar, S., \& Reilly, P. (2018). Is social media bad for mental health and wellbeing? Exploring the perspectives of adolescents. Clinical child psychology and psychiatry, 23(4), 601-613.

Parry, E., \& Urwin, P. (2011). Generational differences in work values: A review of theory and evidence. International Journal of Management Reviews, 13(1), 79-96. doi:10.1111/j.1468-2370.2010.00285.x

Pearl, R. L. (2020). Weight Stigma and the "Quarantine15 ". Obesity.

Perloff, R. M. (2014). Social media effects on young women's body image concerns: Theoretical perspectives and an agenda for research. Sex Roles, 71(11-12), 363377.

Pitron, V., Alsmith, A., \& Vignemont, F. (2018). How do the body schema and the body image interact? Conscious Cogn, 65, 352-358.

Prensky, M. (2001). Digital natives, digital immigrants part 1. On The Horizon, 9(5), 1-6.

Ravalli, M. J., \& Paoloni, P. (2016). Global Kids Online Argentina: Research study on the perceptions and habits of children and adolescents on the use of technologies, the internet and social media.

Ricciardelli, L. A., McCabe, M. P., Williams, R. J., \& Thompson, J. K. (2007). The role of ethnicity and culture in body image and disordered eating among males. Clinical psychology review, 27(5), 582-606.

Rodgers, R. F., \& Melioli, T. (2016). The relationship between body image concerns, eating disorders and internet use, part I: A review of empirical support. Adolescent Research Review, 1(2), 95-119.

Rodgers, R.F., Slater, A., Gordon, C.S., McLean, S.A., Jarman, H.K., Paxton, S.J. (2020). A Biopsychosocial Model of Social Media Use and Body Image Concerns, Disordered Eating, and Muscle-Building Behaviors among Adolescent Girls and Boys. Journal of Youth and Adolescence, 49(2), 399-409.

Sadibolova, R., Ferre, E. R., Linkenauger, S. A., \& Longo, M. (2019). Distortions of perceived volume and length of body parts. Cortex, 111, 74-86.

Sanday, J., Scappatura, M. L., \& Rutsztein, G. (2020). Impacto de la pandemia por COVID-19 en los hábitos alimentarios y la Imagen Corporal. In XII Congreso 
Internacional de Investigación y Práctica Profesional en Psicología. XXVII Jornadas de Investigación. XVI Encuentro de Investigadores en Psicología del MERCOSUR. II Encuentro de Investigación de Terapia Ocupacional. II Encuentro de Musicoterapia. Facultad de Psicología-Universidad de Buenos Aires.

Schaefer, L. M. (2017). Development and validation of the sociocultural attitudes towards appearance questionnaire-4-revised (SATAQ-4R. Int J Eat Disord, 50(2), 104-117.

Solano, A. C., \& Perugini, M. L. L. (2019). Perfiles Diferenciales de Usuarios de Internet, Factores de Personalidad, Rasgos Positivos, Síntomas Psicopatológicos y Satisfacción con la Vida. Revista iberoamericana de diagnóstico y evaluación psicológica, 4(53), 79-90.

Stronge, S., Greaves, L. M., Milojev, P., West-Newman, T., Barlow, F. K., \& Sibley, C. G. (2015). Facebook is linked to body dissatisfaction: Comparing users and nonusers. Sex Roles, 73(5-6), 200-213.

Taylor, P., \& Keeter, S. (Eds.). (2010). Millennials: A portrait of generation next. Washington, DC: Pew Research Center. Retrieved from http:/www.pewsocialtrends.org/files/2010/10/millennia ls-confident-connected-open-to-change.pdf

Thompson, J. K., \& Stice, E. (2001). Thin-ideal internalization: Mounting evidence for a new risk factor for body-image disturbance and eating pathology. Current directions in psychological science, 10(5), 181183.

Turner, A. (2015). Generation Z: Technology and social interest. The journal of individual Psychology, 71(2), 103-113.

Van de Grift, T. C., Cohen-Kettenis, P. T., Elaut, E., De Cuypere, G. R. E. T. A., Richter-Appelt, H., Haraldsen, I. R., \& Kreukels, B. P. (2016). A network analysis of body satisfaction of people with gender dysphoria. Body Image, 17, 184-190.Shrager, J., \& Langley, P. (Eds.) (1990). Computational models of scientific discovery and theory formation. San Mateo, CA: Morgan Kaufmann. 